\title{
Os Tipos do Herbário do Instituto Nacional de Pesquisas da Amazônia - I
}

\author{
Marlene Freitas da Silva (*) \\ Instituto Nacional de Pesquisas \\ da Amazônia
}

\begin{abstract}
SINOPSE
O trabalho é uma primeira divulgação de 42 Tipos da Amazônia disponíveis no herbário do INPA, ilustrado com fotografias das espécies que não tenham sido desenhadas ou mesmo fotografadas pelo autor.
\end{abstract}

\section{INTRODUÇÃo}

Nosso trabalho refere-se apenas ao material existente no herbário enquadrando cada Tipo em sua categoria, conforme as normas previstas pelo "International Code of Botanical Nomenclature" (1961), confrontando-o com a obra, onde tenha sido publicada a espécie.

Como a fonte bibliográfica no que diz respeito às indicações sôbre o material é muitas vêzes suscinta, adotamos as normas de Travassos (1962-1965:240), transcrevendo os dados de coleta constantes nas etiquetas de herbário, pois muito freqüentemente tais obserções de muito valor para os sistematas, não se encontram na bibliografia original. Por outro lado, confirmamos o que observou aquêle mesmo autor quando se referiu ao número citado, dizendo que muitas vêzes encontra-se na publicação original um número como se fôsse do coletor, quando na verdade trata-se do número de registro do Herbário.

No que se refere à ilustração, subsídio de grande importância para a identificação de uma especie, como alguns autores deixaram de fazê-lo, achamos que seria de grande utilidade ilustrarmos nosso trabalho com a fotografia do material Tipo disponível em nosso herbário.
Outro cuidado que tivemos foi o de localizar os herbários onde se encontram exemplares Tipo de nossa coleção, indicando abreviadamente no texto, de acôrdo com o "Index Herbariorum" 4 a ed. (1959), a instituição onde se encontra o referido exemplar. Assim :

$$
\begin{aligned}
& \text { INPA }=\text { Instituto Nacional de Pesquisas } \\
& \text { da Amazônia } \\
& \text { MG = Museu Paraense Emílio Goeldi } \\
& \text { IPEAN = Instituto de Experimentações } \\
& \text { Agro-Pecuárias do Norte } \\
& \mathrm{RB}=\text { Jardim Botânico do Rio de Ja- } \\
& \text { neiro } \\
& \mathrm{HB}=\text { Herbário Bradeanum } \\
& \text { SP = Instituto de Botânica de São } \\
& \text { Paulo } \\
& \mathrm{G}=\text { Conservatoire et Jardin Bota- } \\
& \text { nique de Geneve } \\
& \mathrm{K}=\text { Royal Botanical Garden } \\
& \text { MVM }=\text { Museu de História Natural de } \\
& \text { Montevidéo } \\
& \text { MTCH }=\text { University Herbarium of Michi- }
\end{aligned}
$$

Da presente lista constam 42 Tipos pertencentes a 20 Famílias, e os exemplares vis tos per nós foram os seguintes :

(*) - Bolsista do Conselho Nacional de Pesquisas. 
BIGNONIACEAE

- Pleonothoma dendrotricha Sandw. INPA 2211; 2095

- Tynanthus igneus Barb. Rodr. INPA 27761

BOMBACACEAE

- Catostemma albuquerquei Paula INPA 20551; 15827

- Catostemma milanezii Paula INPA 20548; 20590

CONVOLVULACEAE

- Quamoclit rodriguesii J. I. Falcão INPA 10862

DICHAPETALACEAE

- Gonypetalum lanceolatum D u k e INPA 9038

- Tapura singularis Ducke INPA 16528

EBENACEAE

- Diospyros egleri Pires et Cavalcante INPA 13872

- Diospyros manausensis P. Cavalcante INPA 5516; 842

ELAEOCARPACEAE

- Sloanea bracteosa Ducke INPA 15279

- Sloanea granulosa Ducke INPA 15282

- Sloanea longipes Ducke INPA 15028

ERYTHROXYLACEAE

- Erythroxylum albertianum Kuhlm. et W Rodr.

INPA 83

\section{EUPHORBIACEAE}

- Dalechampia olympiana Kuhlm. et W. Rodr.

INPA 225

GUTTIFERAE

- Caraipa melhemiana Paula INPA 13552

- Caraipa rodriguesii Paula INPA 16650

- Caraipa valioi Paula INPA 20589

- Lorostemon coelhoi Paula INPA 20583
LAURACEAE

- Dicypellium manausense W. Rodrigues INPA 14219; 17040

LECYTHIDACEAE

- Corytophora alta Knuth INPA 15976

LEG. CAESALP.

- Copaifera piresii Ducke INPA 2571

LEG. PAP.

- Ormosia grossa Rudd. INPA 1291

MELASTOMATACEAE

- Aciotis anomala Brade INPA $1318 ; 50$

- Aciotis ferreirana Brade INPA 2198

- Adelobotrys marginata Brade INPA 3264

- Adelobotrys rachidotricha Brade INPA 2228

- Henriettea williamii Brade INPA 569

- Siphantheropsis williamii Brade INPA 630

MYRTACEAE

- Myrcia grandis Mc Vaugh INPA 8407; 8420

- Psidium submetrale Mc Vaugh INPA 4475

PIPERACEAE

- Piper dactylostigmum Yuncker INPA 1854

- Piper manausense Yuncker INPA 3905; 2239

- Piper plurinervosum Yuncker INPA 1075

RUTACEAE

- Fagara dellomei Albuq. INPA 12891

- Fagara djalma-batistae Albuq. INPA 14778; 20561 Fagara prancei Albuq. INPA 24686

$S A B I A C E A E$

- Phoxanthus manausensis W. Rudrigues INPA 12954 
SAPOT ACEAE

- Ecclinusa bacuri Aubrév. et Pellegr. INPA 1094

- Micropholis rosadinha-brava Aubrév. et Pellegr.

INPA 5548

- Micropholis williamii Aubrév. et Pellegr. INPA 1097

- Richardella manausensis Aubrév. et Pel. legr.

INPA 7305

\section{VOCHYSIACEAE}

- Erisma djalma-batistae Paula

INPA $13472 ; 13657$.

\section{BIGNONIACEAE}

Pleonothoma dendrotricha Sandw.

- Mem. N. Y. Bot. Garden 10 (1): 141 (1968)

Nossa coleção referente à espécie acima consta dos Parátipos (INPA 2095, 2211). com exemplares nos herbários do INPA, MG e $\mathrm{K}$.

O material de referência INPA 2095 Paratipo, consta em nosso herbário com os seguintes dados de coleta : Proc. Amazonas, Manaus, Campo Vieiralves, Adrianópolis/Leg. J. Chagas, s.n. / Em 7.X.55 / Obs. Trepadeira com frutos planos; t. firme, arenoso, capoeira aberta.

0 material de referência INPA 2211 possui dois exemplares em nosso herbário, com os seguintes dados de coleta: Proc. Amazonas, Manaus, estrada antiga de S. Raimundo / Leg. J. Chagas, s.n. / Em 24.X.55 / Obs. Trepa deira com frutos verdes; $t$. firme, capoeira fe chada, solo arenoso.

Nota - Embora não referido, nossa coleção consta de material com flôres e frutos. (Foto 1).

Tynanthus igneus Barb. Rodr.

- Vellozia, : 50-51; tab. X. (1891).

Embora não tenha o autor feito referên. cias à coleção Tipo em seu trabalho, consideramos os fragmentos encontrados por William Rodrigues entre as páginas $372-373$ do volume $\mathrm{n}^{\circ} 5$ de Adansonia (1895), como frag-

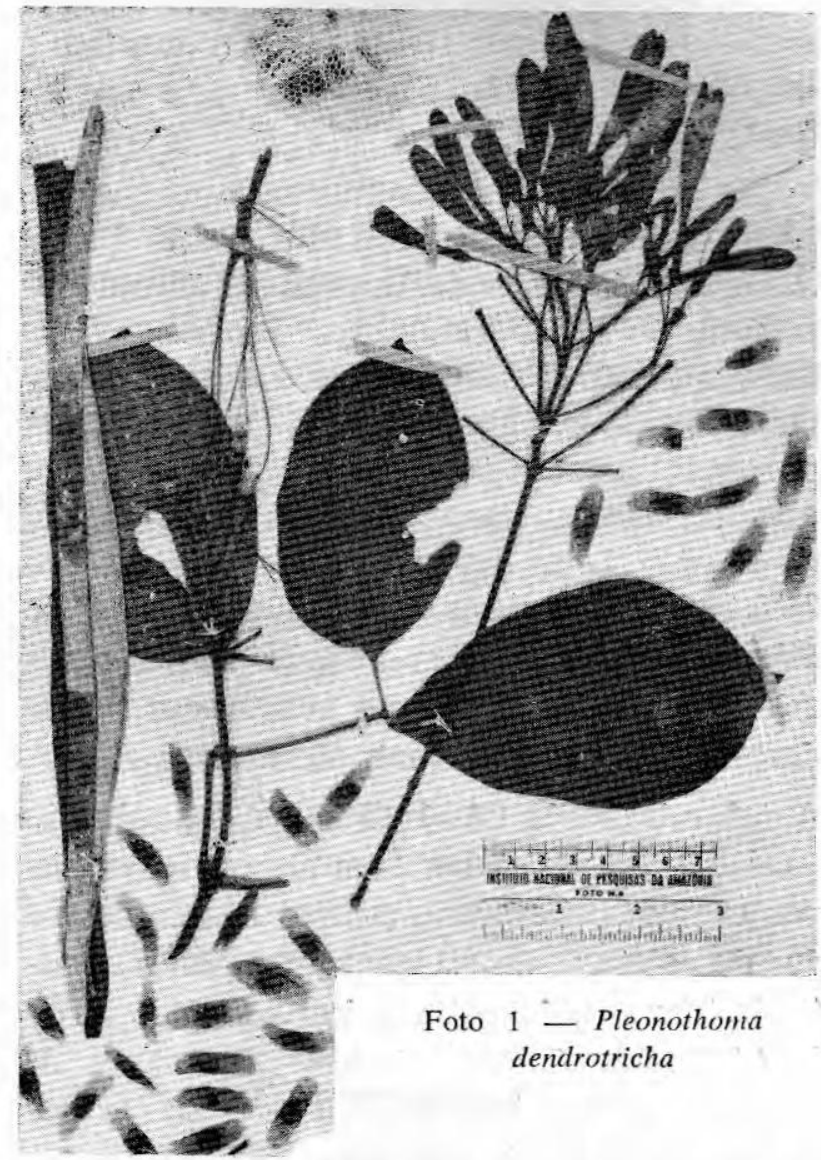

mento Tipo da espécie acima, por corresponder exatamente, com riqueza de detalhes. àquele utilizado por Barbosa Rodrigues para ilustração de sua nova espécie. O exemplar foi incluído no herbário do INPA com os seguintes dados: Herb. INPA 27761 / N. cient. Tynanthus igneus Barb. Rodr. = Pyrostegia cinerea Bur. / Proc. Amazonas, Manaus, margem do rio Negro / Leg. B. Rodrigues, s.n. e data de coleta .

NотA - A espécie acima foi considerada por Burret como sinônimo de Pyrostegia cinerea, in Mart. Fl. Brasil. 4 (1) : 1862 - 1863.

\section{BOMBACACEAE}

Catostemma albuquerquei Paula

- Ciência e Cultura. Soc. Bras. Prog. da Ciência, 20(2): 313. (1968).

- Idem, 21(4) : 702; figs. 16, 17, 18, 19, 27 (1969). 
A espécie acima consta em nossa coleção do Holótipo, Isótipos (INPA 20551), Parátipo e Isoparátipos (INPA 15827), assim consignados :

A coleção INPA 20551, conta com exemplares nos herbários do INPA, Inst. Ciênc. Biológicas da Univ. de Brasília, e, Jardin Botanique de l'Etat (Bélgica). Em nosso herbário, além do Holótipo temos mais três exemplares Isótipos, igualmente etiquetados, com os seguintes dados de coleta : Proc. Amazonas. estrada Manaus-Itacoatiara, $\mathrm{km} \mathrm{104,} \mathrm{picada}$ do INPA / Leg. Byron et Elias, 67-44 / Em 9.V.1967 / Obs. Árvore de $30 \mathrm{~m} \mathrm{x} 35 \mathrm{~cm}$ de diâmetro; fuste de $22 \mathrm{~m}$. Botões florais amarelados. Frutos maduros, castanhos, veludosos. Solo argiloso, mata de t. firme.

A coleção INPA 15827, consta além do Parátipo, de mais dos exemplares Isoparátipos em nosso herbário, com os seguintes dados de coleta: Proc. Amazonas, Manaus, estrada Manaus-Itacoatiara, $\mathrm{km} \mathrm{106,} \mathrm{lado} \mathrm{direi-}$ to / N.V. Mamãorana / Leg. W. Rodrigues, A Loureiro, 7164 / Em 14.IX.65 / Obs. Árvore de $25 \mathrm{~m} \times 45 \mathrm{~cm}$ de diâmetro. Frutos maduros, amarelo-ferrugíneos. Copa mudando a folhagem. Casca lisa, rajada Semente com forte odor de estearina. Mata de $t$. firme, solo argiloso.

\section{Catostemma milanezii Paula}

- Ciência e Cultura. Soc. Bras. Prog. da Ciência, 20(2)：313. (1968)

- Idem, 21(4) : 702; figs. 14, 15 . (1969)

A coleção referente à espécie acima consta em nosso herbário do Holótipo, Isótipos (INPA 20584) e Parátipo (INPA 20590), cujos exemplares estão assim distribuídos :

Coleção INPA 20584, com dois exemplares em nosso herbário, sendo um Holótipo, e outro Isótipo, contém os seguintes dados de coleta: Proc. Amazonas, Manaus, Reserva Florestal Ducke, Quadra 2 / N. V. Falso cardeiro / Leg. J. Elias, 401 / Em 27.VI.67 / Obs. Árvore de $25 \mathrm{~m}$ x $45 \mathrm{~cm}$ de diâmetro; fuste de 20m. Solo argiloso, bosque. Frutos deiscentes. Resina creme, elástica, no alburno.

Coleção INPA 20590 consta apenas do exemplar Parátipo, com os seguintes dados de coleta : Proc. Amazonas, Manaus, Reserva Florestal Ducke / Leg. J.E. de Paula, 393 / Em 5.V.67 / Obs. Árvore de $20-25 \mathrm{~m} \times 38 \mathrm{~cm}$ de diâmetro; frutificação jovem.

\section{CONVOLVULACEAE}

\section{Quamoclit rodriguesii J. I. Falcão}

- Publ. INPA 25 (Botânica) : 9-10; Fig. 2 (1968) .

Nossa coleção referente à espécie acima consta do Holótipo e do Isótipo (INPA 10862), com um exemplar no herbário do Jardim Botânico do Rio de Janeiro (RB 125.241).

O material de referência INPA 10862 está representado em nosso herbário por dois exemplares, o Holótipo e um Isótipo, com os seguintes dados de coleta: Proc. Território Federal de Rondônia, Forte Principe da Beira, Rio Guaporé, Porto Acre / Leg. W. Rodrigues, D. Coêlho, 4296 / Em 13.I.1962 / Obs. Campo, solo areno-argiloso, úmido. Flôres alaranjadas com listras branco-avermelhadas. Arbusto de $1 \mathrm{~m}$.

\section{DICHAPETALACEAE}

\section{Gonypetalum lanceolatum Ducke}

- Arch. Jard. Bot. Rio de Janeiro VI : 43 (1933).

O exemplar INPA 9038 é Isosíntipo, e consta apenas uma exsicata em nosso herbário, com os seguintes dados de coleta: Proc. Amazonas, Manaus, Cachoeira Grande do Tarumã / Leg. A. Ducke, s.n. / Em 2.II.1930 / Obs. Árvore média; flôres amarelo-brancacento, turvo.

Nota - Atualmente, seg. Rizzini in Rev. Bras. Biol. XII : 105 (1952), a espécie acima passou a sinônimo de Tapura lanceolata (Ducke) Rizzini. (Foto 2). 


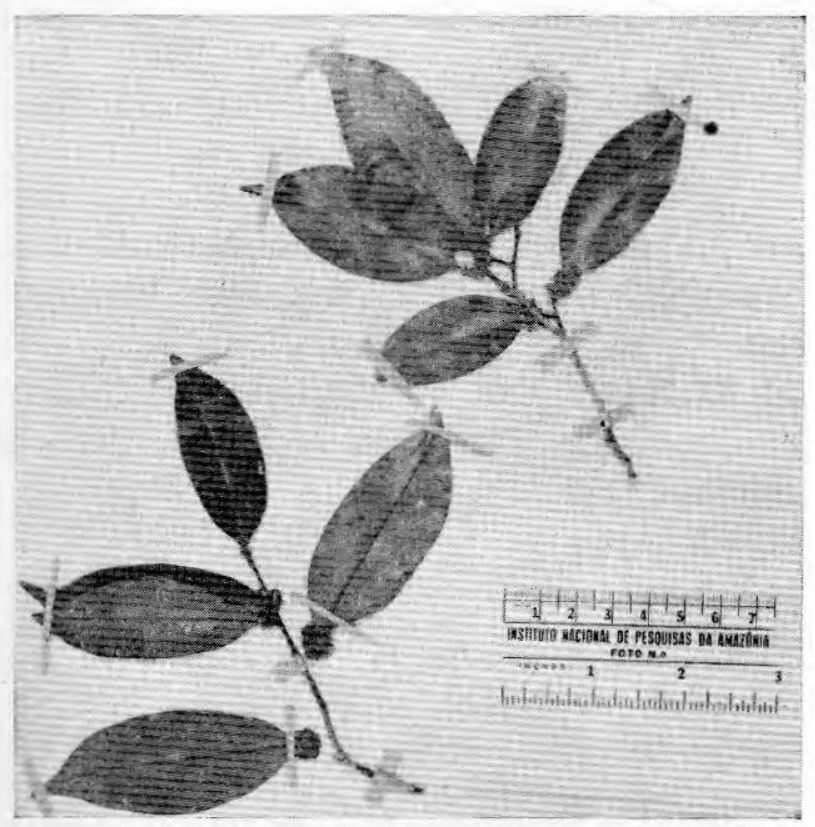

Foto $2-$ Gonypetalum lanceolatum

Tapura singularis Ducke

- Trop. Woods 90: 21 (1947).

O exemplar INPA 16528 é Isosíntipo, do qual possuimos apenas um exemplar com os seguintes dados na etiqueta: Proc. Pará, Belém, Bosque Municipal / Leg. A. Ducke, 1930 / Em 4.IV.1946 / Obs. Árvore pequena; flor quando nova de côr branca, passando ràpidamente para o amarelo-alaranjado. (Foto 3)

\section{EBENACEAE}

Diospyros egleri Pires et Cavalcante

- Bol. Mus. Par. Emílio Goeldi, Bot. 9 : 3-4; est. II e V (1960).

A coleção INPA 13872 Parátipo, consta de apenas um exemplar em nosso herbário, com a seguinte etiqueta de coleta: Proc. Amazonas, Rio Negro, Ilha das Flores / Leg. P. Cavalcante, 770 / Em 21.I.60 / Obs. Arvoreta de $6-8 \mathrm{~m}$ de altura, na margem do rio; flôres branco-amareladas, cálice verde-cinza.
Diospyros manausensis $\mathrm{P}$. Cavalcante

- Bol. Mus. Par. Emílio Goeldi, N. série Bot. 22 : 2-3; Figs. 6,7 e 8 (1966).

Nossa coleção referente à espécie acima consta do Holótipo (INPA 5516) e do Parátipo (INPA 842), com exemplares doados ao herbário do MG (Belém), registrados sob os números 24938 e 24940 , respectivamente.

A coleção INPA 5516 com apenas um exemplar em nosso herbário, possui as seguintes indicações de coleta: Proc. Amazonas, Manaus, estrada do Aleixo, $\mathrm{km} 12 / \mathrm{Leg}$. L. Coêlho, s.n. / Em 19.IV.1957 / Obs. Árvore de $15 \mathrm{~m}$ de altura; mata de t. firme, solo argiloso; frutos amarelo-esverdeados.

Da coleção INPA 842 possuimos apenas um exemplar no qual consta os seguintes dados de coleta : Proc. Amazonas, Manaus, cabeceira do igarapé da Cachoeira Alta do Ta-

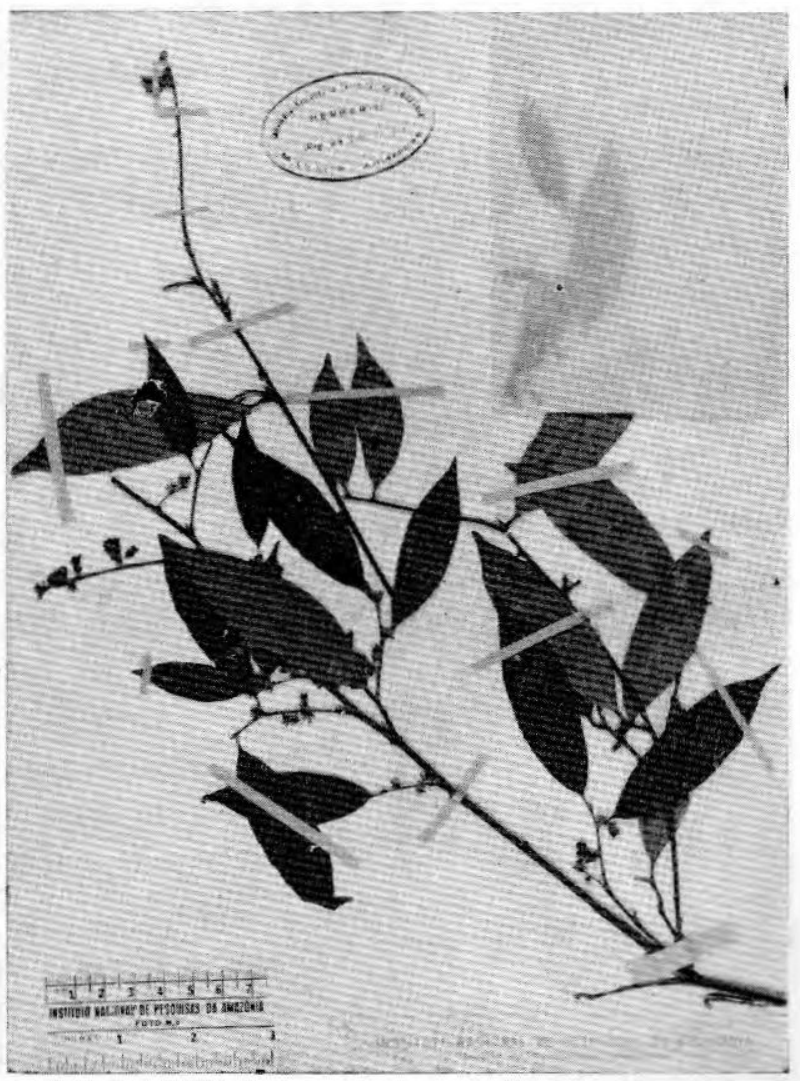

Foto 3 - Tapura singularis 
rumã. / Leg. J. Chagas, s.n. / Em 2.III.1955 / Obs. Arvoreta de $5 \mathrm{~m}$ de altura: mata de t. firme; frutos verdes.

\section{ELAEOCARPACEAE}

\section{Sloanea bracteosa Ducke}

- Arch. Inst. Biol. Vegetal do Rio de Janeiro 2 (2) : 168. (1935).

O exemplar INPA 15279 é Isosíntipo, e consta de apenas um exemplar em nosso herbário, com a seguinte etiqueta : Proc. Amazonas, Manaus, estrada do Aleixo, $\mathrm{km} 9$. Leg. A. Ducke, s.n. / Fl. 9.XII.1932; Fr. 18.IV.1933 / Obs. Árvore mediana, flor rósea com estames bem amarelos; mata de $t$. firme.

Nota - Atualmente, esta espécie é sinônimo de Sloanea nitida G. Don. (Foto 4).

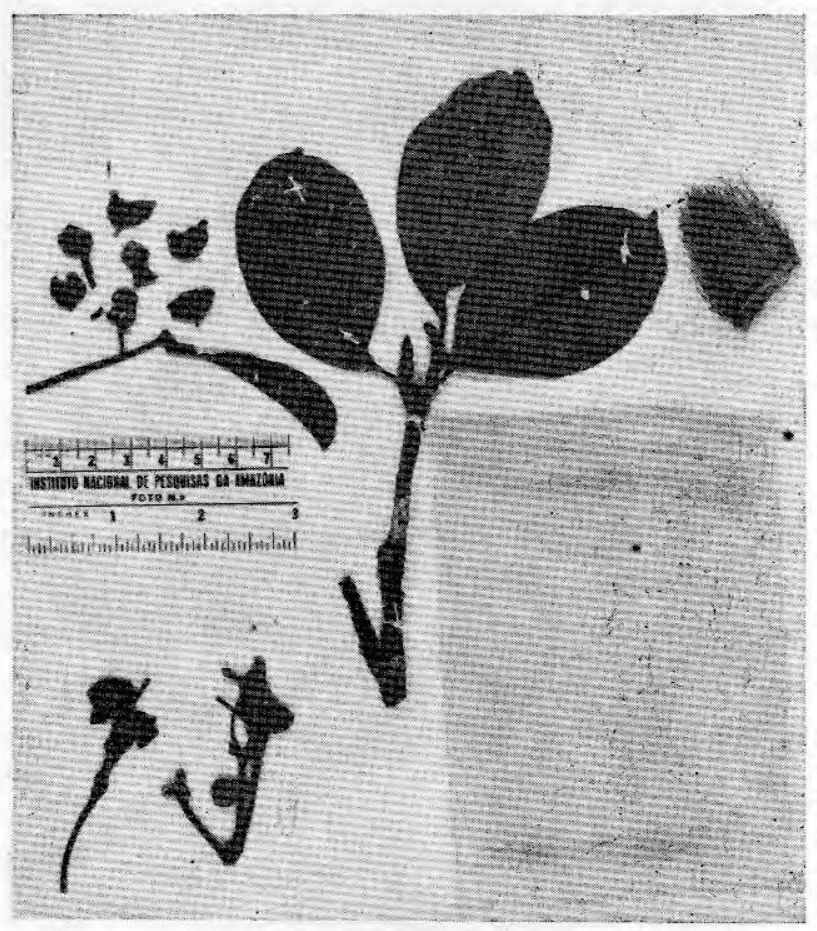

Foto $4-($ Sloanea bracteosa $)=S$. nitida

\section{Sloanea granulosa Ducke}

- Bol, Tec. Inst. Agron. Norte, 19 : 13. (1950)

O exemplar INPA 15282 é Isosíntipo com apenas uma amostra em nosso herbário, com os seguintes dados de coleta : Proc. Pará, Belém, Catu / Leg. A. Ducke, 1656 / Em .... 21.XI.1944 / Obs. Ârvore altíssima, sapopema muito grande; mata de t. firme; flôres cremes. (Foto 5).

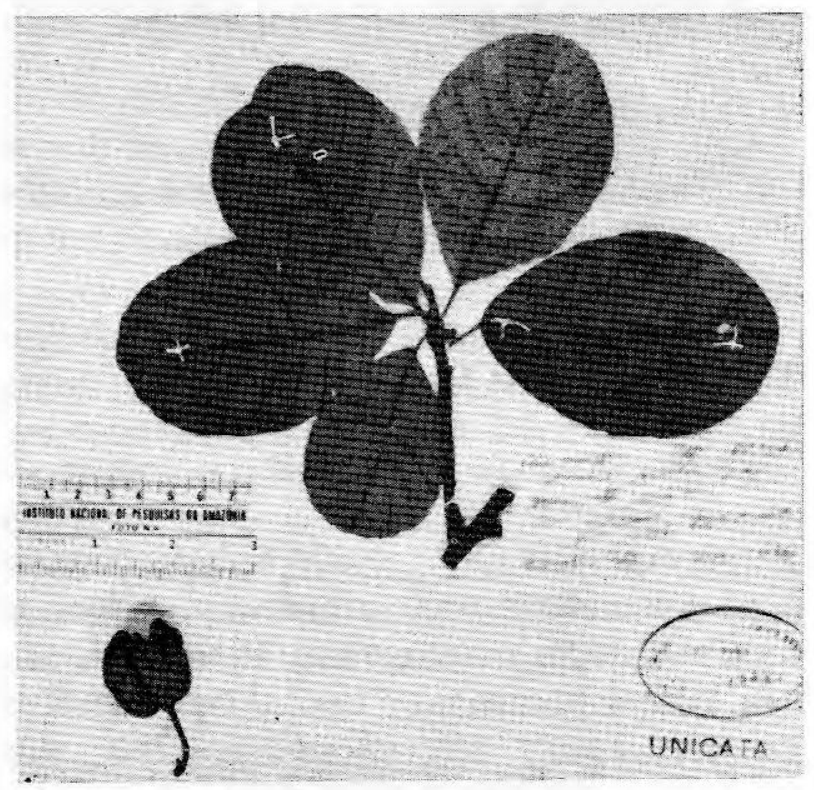

Foto 5 - Sloanea granulosa

\section{Sloanea longipes Ducke}

- Arch. Inst. Biol. Vegetal, Rio de Janeiro 2 (2) : 166 (1935) .

O exemplar INPA 15028 é Isosíntipo, e figura em nosso herbário como unicata, com os seguintes dados de coleta : Proc. Pará, Breves, rio Macujubim / Leg. A. Ducke, s.n. Em 9.VII. 1923.

NotA - Atualmente a espécie acima é sinônimo de Sloanea floribunda Spr. ex Bth. (Foto 6). 


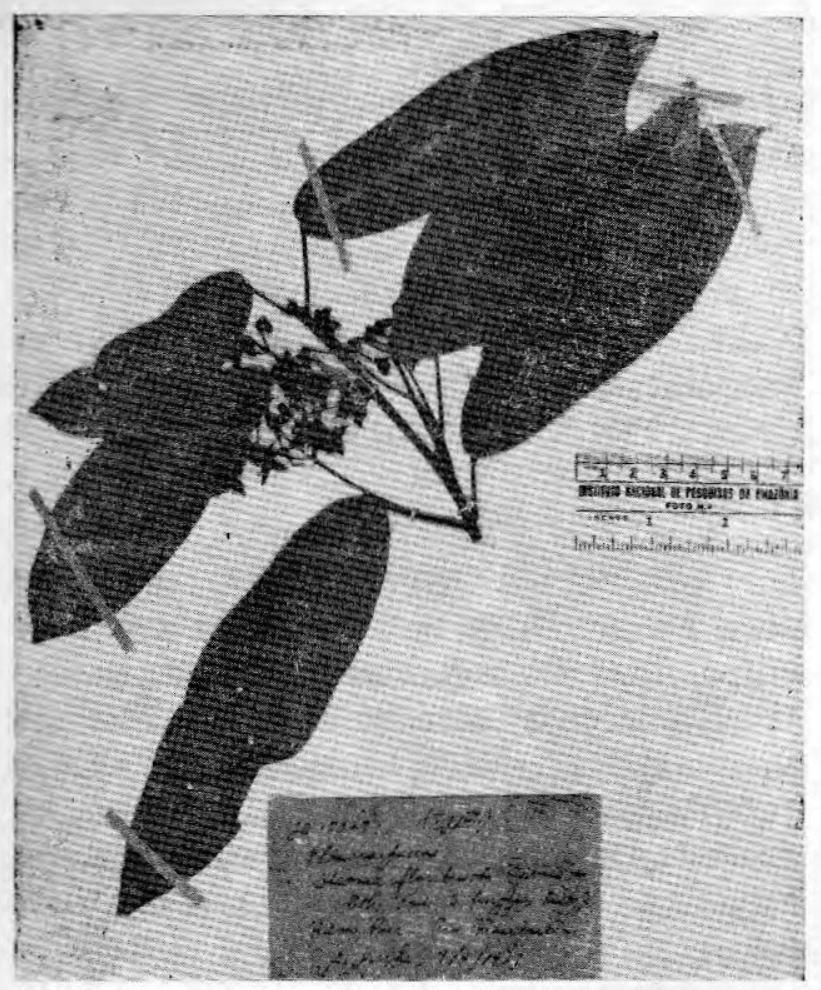

Foto $6-($ Sloanea longipes $)=S$. floribunda

\section{ERYTHROXYLACEAE}

Erythroxylum albertianum J. G. Kuhlmann et W. Rodr.

- Bol. INPA, Bot. 5 : 3-4; foto $\mathrm{s} / \mathrm{n}$. (1957)

O exemplar INPA 83 é Isosíntipo, com uma exsicata em nosso herbário, e uma amostra no herbário do MG (Belém), com os seguintes dados de coleta: Proc. Amazonas, Manaus, Igarapé da Cachoeira Alta do Tarumã / Leg. W. Rodrigues, s.n. / Em 12.IX.1955 / Obs. Árvore de 3m altura; flores esverdeadas; t. firme.

\section{EUPHORBIACEAE}

Dalechampia olimpiana Kuhlm. et W. Rodr.

- Bol. INPA, Bot. 5 : 1-3; foto s/n. (1957)

A coleção INPA 225 figura em nosso herbário como Isosíntipo, da qual possuimos duas exsicatas com as seguintes indicações de coleta : Proc. Amazonas, Manaus, cabeceira do Igarapé do Buião, Colônia Campo Sales / Leg. J. Chagas, s.n. / Em 15.X.1954 / Obs. Trepadeira escandente; flôres esverdeadas; t. firme.

\section{GUTTIFERAE}

Caraipa melhemiana Paula

- Ciência e Cultura. Soc. Bras. Prog. Ciência 20 (2) : 133 (1968).

- Idem..., 21 (4) : 170; Figs. 7, 8, 9, 10, 11. (1969) .

A coleção INPA 13552, Holótipo, consta de apenas uma exsicata em nosso herbário, com as seguintes indicações de coleta : Proc. Ter. Amapá, Serra do Navio / Leg. N.Y. Bot. Garden, 51190 / Em 25.IX.1961 / Obs. In bud, tree $30 \mathrm{~m}$ high $\times 1,5 \mathrm{~m}$ diam.; buds yellowgreen; stamens white upland plant.

\section{Caraipa rodriguesii Paula}

- Ciência e Cultura. Soc. Bras. Prog. Ciência. 20 (2) : 313 (1968) .

- Idem..., 21 (4) : 712; Figs. 13, 14, 15 e 16. (1969).

A coleção INPA 16650 possui além do Holótipo sete exemplares Isótipos, com etiquetas idênticas, contendo os seguintes dados de coleta: Proc. Amazonas, Manaus, AM-1, km 175, picada 23 , a $740 \mathrm{~m}$ da estrada / Leg. W. Rodrigues, D. Coêlho, 7312 / Em 27.XI.65 / Obs. Árvore de $25 \mathrm{~m} \times 30 \mathrm{~cm}$ de diâmetro. Igapó. Flôres brancas com estames amarelos. Látex amarelo.

Caraipa valioi Paula

- Ciência e Cultura. Soc. Bras. Prog. Ciência, 20 (2) : 313 (1968).

— Idem..., 21 (4) : 714; Figs. 17, 18, 19, 20, 21 e 22 . (1969). 
A coleção INPA 20589 consta em nosso herbário do Hólotipo, e mais cinco exemplares Isótipos, com etiquetas idênticas, contendo os seguintes dados de coleta: Proc. Amazonas, Manaus, Reserva Florestal Ducke, picada 2 do levantamento base / Leg. L. Coêlho et Elias, 419 / Em 30.VI. 1967 / Obs. Árvore de 30m de altura por $45 \mathrm{~cm}$ de diâmetro; fuste de $25 \mathrm{~m}$; flôres avermelhadas; solo argiloso; mata de t. firme; frutos coletados em 18.IX. 1967.

\section{Lorostemon coelhoi Paula}

- Ciência e Cultura. Soc. Bras. Prog. Ciência, 20 (2) : 313 (1968).

— Idem..., 21 (4) : 708; Figs. 1, 2, 3, 4, 5 e 6 . (1969).

Nossa coleção INPA 20783 possui aiém do Holótipo um exemplar Isótipo com etiquetas idênticas, cujos dados de coleta são os seguintes : Proc. Amazonas, Manaus, Reserva Florestal Ducke, estrada que dá acesso à Reserva, lado esquerdo, próximo ao antigo portão. Leg. L. Coêlho et Elias, 420 / Em 21.VI.1967 / Obs. Árvore de $22 \mathrm{~m} \times 25 \mathrm{~cm}$ diâmetro; fuste de $18 \mathrm{~m}$. Frutos bastante adiantados, ainda verdes, angulosos-sulcados longidutinalmente. Cinco feixes estaminíferos persistentes. Sépalas cônicas, imbricadas. Brácteas cônico-agudas, imbricadas. Resina creme. Árvore próxima ao Fenológico no 49. Solo argiloso, bosque. Período de frutificação: 14 meses.

\section{LAURACEAE}

Dicypellium manausense W. Rodr.

- Bol. INPA, Bot. 25 : 3-5; Fig. 1 (a, b, c, d e, f, g, h). (1968).

Nossa coleção referente à espécie acima consta do Holótipo, Isótipos (INPA 14219), Parátipo e Isoparátipos (INPA 17040), assim distribuídos :
A coleção INPA 14219 contém exemplares nos herbários do INPA e NY. Em nosso herbário contamos além do Holótipo com três Isótipos, cujos dados de coleta são os seguintes : Proc. Amazonas, Manaus, Reserva Florestal Ducke, margem da estrada. / N.V. Louro preto / Leg. W. Rodrigues, 5546 / Em 28.IX.1963 / Obs. Mata de t. firme, solo argiloso, amarelo. Árvore de $10 \mathrm{~m} \times 10 \mathrm{~cm}$ de diâmetro. Flôres brancas. Frutos roxos com o cálice vermelho. Ocorrência ocasional.

A coleção INPA 17040 com exempiares nos herbários do INPA, NY e G, consta em nosso herbário além do Parátipo de um Isoparátipo, com os seguintes dados de coleta : Proc. Amazonas, Manaus, Reserva Florestal Ducke, Q. XIV. / N.V. Louro / Leg. W. Rodrigues, D. Coêlho, 7735 / Em 29.IV.1966 / Obs. Arvoreta de $4 \mathrm{~m}$ com frutos roxos, maduros, com pedicelo vermelho. Mata de $t$. firme, solo argiloso.

\section{LECYTHIDACEAE}

\section{Corytophora alta Knuth}

- Das Pflanzenreich 105, heft (219, 219a. 219b) : 51. Fig. 10. (1956).

O exemplar INPA 15976 é Isótipo, e consta em nosso herbário de apenas um exemplar, com os seguintes dados de coleta : Proc. Pará, rio Trombetas, Castanhais do Jacaré / Leg. A. Ducke, s.n. / Em 11.I.1927 / Obs. Árvore grande, flor rósea.

\section{LEG. CAESALP.}

\section{Copaifera piresii Ducke}

— Bol. Tec. Inst. Agr. Norte, 36 : 73-74 (1959)

O exemplar INPA 2571 é Isótipo, com apenas uma exsicata em nosso herbário, cuja ficha contém os seguintes dados: Proc. Pará, rio S. Manoel, Pôsto dos índios Caiabi / Leg. J. M. Pires, 3876 / Em 8.I. 1952 / Obs. Arbusto de campo; flor branca-esverdeada. (Foto 7). 


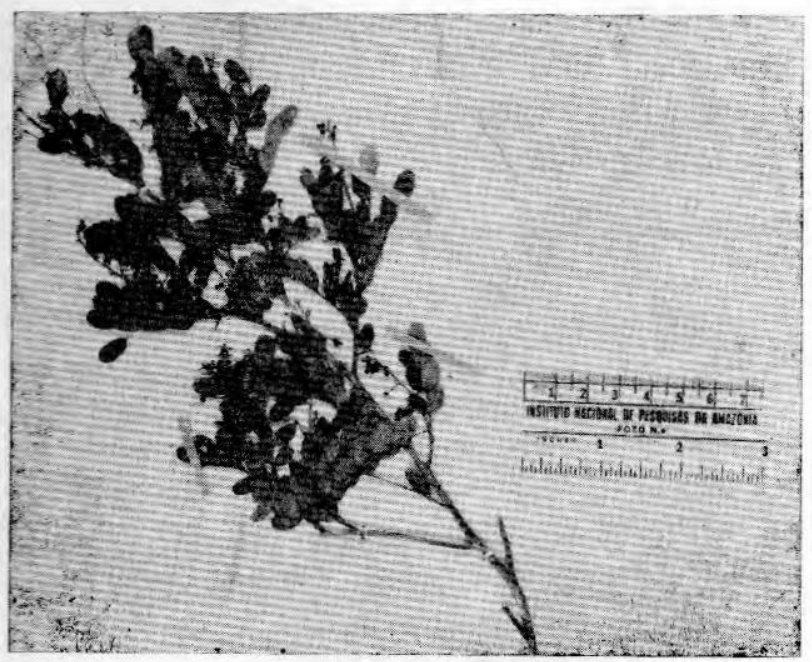

Foto 7 - Copaifera piresii

NoтA - Manuseando material dessa família, chamou-nos a atenção a espécie $\boldsymbol{C}$. minima. Para surprêsa nossa comprovamos que como tal não havia sido publicada. Mais tarde porém, consultando Egler, W. (1963), verificamos tratar-se do Isótipo de C. $p i$ resii Ducke. De tudo conclui-se que o autor, não havendo publicado a espécie em 1954, data da determinação escrita por êle na etiqueta, quando em 1959 publicou-a, usou outra denominação específica.

LEG. PAP.

Ormesia grossa Rudd.

- Contr. United States Herbarium, 32 (5) : 331; Fig. 9 (1965).

A coleção INPA 1291 é Isótipo, com apenas um exemplar em nosso herbário, com os seguintes dados de coleta: Proc. Amazonas, Manaus, Cachoeira Baixa do Tarumã / Leg. W. Rodrigues, s.n. / Em 1.VII.1955 / Obs. Árvore de 8m de altura, com vagens vermelhas, lenhosas, unispermas; sementes bicolores (prêto e vermelho); capoeira grossa da t. firme.
MELASTOMATACEAE

\section{Aciotis anomala Brade}

- Amazonia, INPA Bot. 8: 8; Est. 2 (1958).

Nossa coleção referente à espécie acima consta do Holótipo e Isótipo (INPA 1318), e Parátipo (INPA 50), com exemplares nos herbários do INPA, MG, e IPEAN, assim consignados :

A coleção INPA 1318 possui além do Holótipo um exemplar Isótipo em nosso herbário, e dois exemplares nos herbários do IPEAN e MG respectivamente, com as seguintes indicações de coleta : Proc. Amazonas, Manaus, km 24 da estrada BR-17 / Leg. F. Mello, s.n. / Em 5.VII. 1955 / Obs. Arbusto de $20 \mathrm{~cm}$ em terreno brejado.

A coleção INPA 50 com exemplares nos herbários do INPA, MG e IPEAN, figura em nosso herbário com apenas um exemplar, com as seguintes indicações de coleta : Proc. Amazonas, Manaus, $\mathrm{km} 10$ da estrada BR-17, Campo Sales / N.V. Barba de paca / Leg. J. Chagas, s.n. / Em 30.VIII.1954 / Obs. Arbusto com flôres alvas; fôlhas róseas na face inferior; terreno alagadiço.

Nota - O número que na descrição original é dado como número do coletor, refere-se ao número de registro do INPA.

Aciotis ferreirana Brade

- Amazônia. INPA, Bot. 8 : 10-12; Est. 3 (1958) .

A coleção INPA 2198, consta em nosso herbário do Holótipo, e um exemplar Isótipo com amostras nos herbários do MG e HB. Em nossos exemplares constam os seguintes dados de coleta : Proc. Amazonas, Manaus, Igarapé de S. Raimundo / Leg. J. Chagas, s.n. / Em 21.X.1955 / Obs. Erva rasteira no igapó. 
Adelobotrys marginata Brade

— Amazônia. INPA, Bot. 8 : 14; Est. 5 (1958)

A coleção referente à espécie acima consta de Holótipo, Isótipos (INPA 3264), e Parátipo (INPA 961), com exemplares nos herbários do INPA e MG.

Da coleção INPA 3264, consta em nosso herbário o Holótipo e dois exemplares Isótipos com os seguintes dados de coleta : Proc. Amazonas, Manaus, Igarapé de Santa Maria / Leg. D. Coêlho, s.n. / Em 4.I.1956 / Obs. Trepadeira escandente na mata de t. firme, solo arenoso, úmido.

A coleção INPA 961 figura em nosso herbário com apenas um exemplar, com os seguintes dados de coleta: Proc. Amazonas, Manaus, Igarapé do Bindá / Leg. J. Chagas, s.n. / Em 18.IV.1955 / Obs. Trepadeira com caule e fôlhas densamente ferrugíneo-tomentosas no dorso; frutos verdes.

\section{Adelobotrys rachidotricha Brade}

- Amazônia. INPA, Bot. 8 : 12; Est. 4 (1958)

A coleção de referência INPA 2228 Holótipo, com exêmplares nos herbários do INPA, IPEAN e MG, figura em nosso herbário com apenas um exemplar, possuindo os seguintes dados de coleta: Proc. Amazonas, Manaus, margem do Igarapé de Santa Maria / Leg. J. Chagas, s.n. / Em 26.X.1955 / Obs. Trepadeira com flôres alvas na mata de t. firme, solo arenoso.

\section{Henriettea williamii Brade}

- Amazônia. INPA, Bot. 8 : 17; Est. 6 (1958)

Da coleção INPA 569 possuimos apenas um exemplar Holótipo, com os seguintes dados de coleta: Proc. Território Federal do Rio Branco, Serra do Maturuca, margem do igarapé Urucazinho / Leg. W. Rodrigues, 95 Em 19.XI. 1954 / Obs. Arbusto de 2,5m em terreno úmido; flôres alvas, estames lilazes.

Siphantheropsis wiliamii Brade

- Amazônta. INPA, Bot. 8 : 4; Est. 1 (1958)

Da coleção INPA 630 só possuumos um exemplar, o Holótipo, com os seguintes dados de coleta: Proc. Território Federal do Rio Branco, margem do igarapé Canan / Leg. W. Rodrigues, 142 / Em 22.XI.1954 / Obs Erva com flôres alvas e encarnadas.

NotA - Seg. Wurdack, a espécie acima é a mesma Macairea lasiophylla (Bth.) Wurdack.

\section{MYRTACEAE}

Myrcia grandis Mc Vaugh

- Mem. N. Y. Botanical Garden 18 (2) : 114-115 (1969).

A coleção referente à espécie acima consta do Parátipo e Isoparátipo, de referência INPA 8407 e 8420 respectivamente, com exemplares nos herbários do INPA e MVM. Em nosso herbário possuimos além do Parátipo um exemplar Isoparátipo da coleção INPA 8407, com os seguintes dados de coleta : Proc. Amazonas, Manaus, Enseada Grande, Ponta Negra / Leg. W. Rodrigues, L. Coêlho, 2030 / Em 30.XII.1960 / Obs. Arbusto de 4m; frutos vermelhos; margem do rio.

A coleção INPA 8420 conta com exemplares nos herbários do INPA, SP e MVM, sendo que em nosso herbário existe o Parátipo e um exemplar Isoparátipo, com as seguintes indicações de coleta: Proc. Amazonas, Manaus, Enseada Grande, Ponta Negra / Leg. W. Rodrigues, L. Coêlho, 2042 / Em 3.I.1961 / Obs. Arbusto de $4 \mathrm{~m}$ de altura; flôres alvas; frutos vermelhos; margem do rio. (Foto 8). 


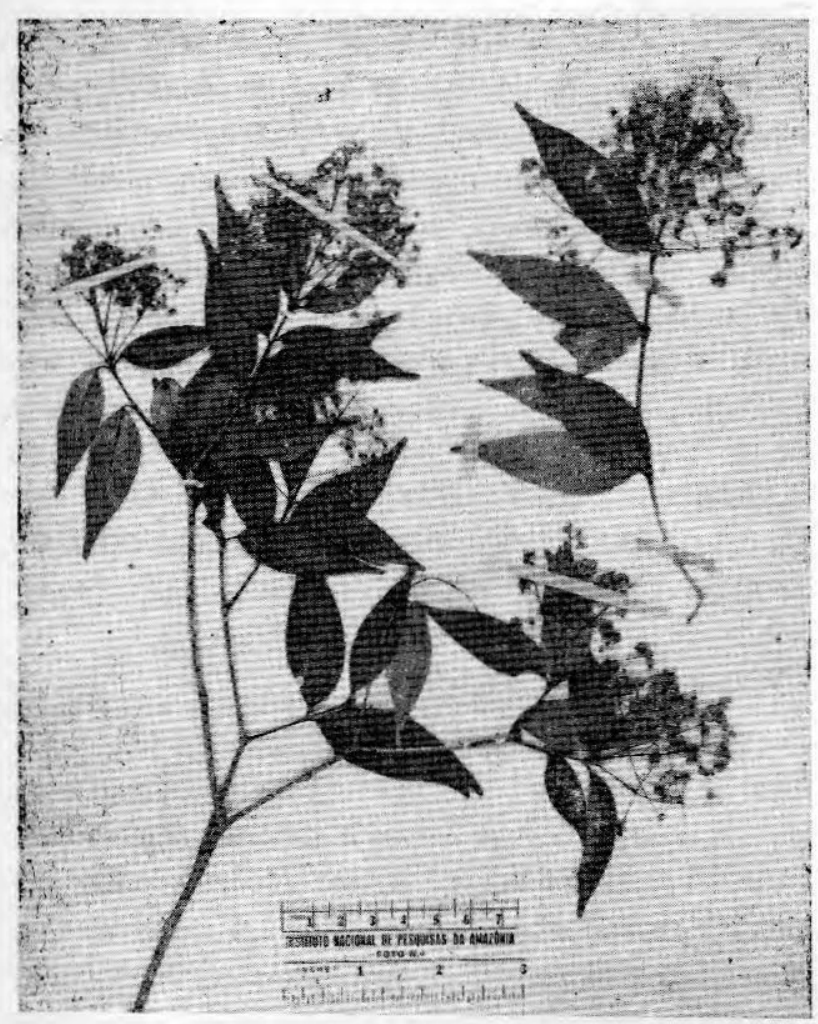

Foto $8-$ Myrcia grandis

Em nosso herbário temos dois Isótipos, com as seguintes observações de coleta : Proc. Amazonas, Manaus, $\mathrm{km} 10$ da rodovia BR-17 / Leg. J. Chagas, s.n. / Em 8.IX.1955 / Obs. Trepadeira na mata de t. firme, de solo arenoso.
Nota - Segundo o autor, o exemplar Holó- tipo está em nossa coleção, porém, o mesmo ainda não nos foi devolvido. Por essa razão nossos exemplares são apenas Isótipos.

Piper manausense Yunker

- Bol. Sec. Agr. Est. S. Paulo, Inst. Botânica 3 : 107; Fig. 93 (1966).

Nossa coleção referente à espécie acima constitui-se do Parátipo e Isoparátipos (INPA 2239 e 3905 ), assim distribuídos :

A coleção INPA 2239 possui exemplares nos herbários do INPA, MG, IPEAN e DPU. Em nosso coleção temos o Parátipo e um Iso-

Psidium submetrale Mc Vaugh

- Mem. N. Y. Botanical Garden 18 (2) : 261-262 (1969).

A coleção de referência INPA 4475 representada em nosso herbário por apenas uma exsicata é Síntipo e possui um exemplar no herbário de Mich. Nosso exemplar contém os seguintes dados de coleta: Proc. Território Federal do Rio Branco, Vila de Normandia / Leg. W. Rodrigues, s.n. / Em 6.XI.1954 / Obs. Arbusto com flôres alvas; terreno de savana. (Foto 9).

\section{PIPERACEAE}

Piper dactylostigmum Yunker

- Bol. Sec. Agr. Est. S. Paulo, Inst. Botânica 3: 35-36; Fig. 29. (1966).

A coleção INPA 1854 conta com exemplares nos herbários do INPA, MG, IPEAN e DPU.

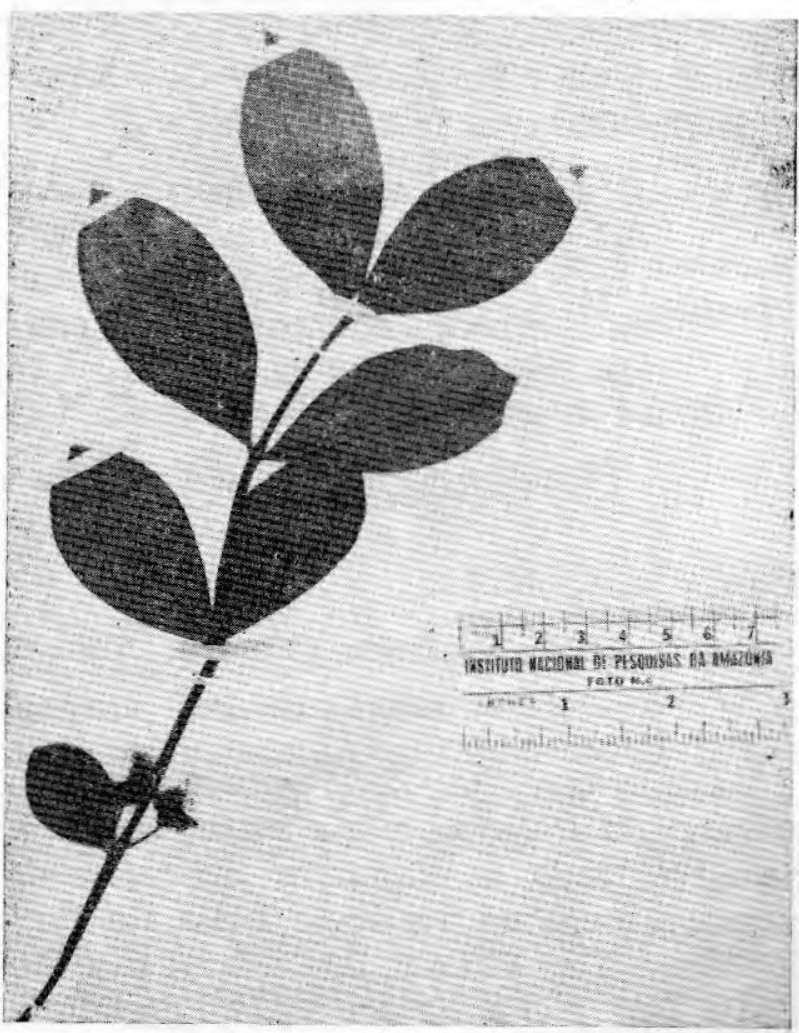

Foto 9 - Psidium submetrale 
parátipo, com as seguintes indicações de coleta : Proc. Amazonas, Manaus, km 30 da BR-17 / Leg. F. Mello, s.n. / Em 26.X.1955 / Obs. Arbusto de $1 \mathrm{~m}$ na mata de $t$. firme, solo arenoso. Inflorescência em espiga.

A coleção INPA 3905 Parátipo, com apenas um exemplar em nosso herbário, possui as seguintes indicações de coleta: Proc. Amazonas, Manaus, Igarapé Mariano / N.V. Pimenta longa brava / Leg. J. Chagas, s.n. / Em 8.VI.1956 / Obs. Mata de t. firme, solo argiloso.

Nota - Na publicação original onde se encontra (IPA), deve ser lido INPA.

\section{Piper plurinervosum Yunker}

- Bol. Sec. Agr. Est. S. Paulo, Inst. Botânica 3: 62-63; Fig. 52 (1966).

Nossa coleção INPA 1075 é Isótipo e consta de duas exsicatas em nosso herbário, com uma amostra no herbário de DPU. Em nossos exemplares constam os seguintes dados de coleta : Proc. Amazonas, Manaus, Cachoeira do Tarumã / N. V. Pimenta longa / Leg. J. Chagas, s.n. / Em 27.v.1955 / Obs. Arbusto de $3 \mathrm{~m}$ altura, na mata de $t$. firme.

NотA - 0 autor em seu trabalho diz que o Holótipo está em nossa coleção porém o mesmo ainda não nos foi devolvido, consequentemente, nossa coleção consta apenas de Isótipos.

\section{RUTACEAE}

Fagara dellomei Albuq.

— Publ. INPA, Botânica 27: 3-4; Tab. 1 (1968) .

A coleção INPA 12891 consta em nosso herbário do Holótipo e três exemplares Isótipos, contendo as seguintes indicações de coleta : Proc. Pará, região do Tiriós, rio Paru do Oeste / Leg. Fittkau, D. Coêlho, s.n. / Em 29.III. 1962 / Obs. Árvore de $24 \mathrm{~m}$ de altura, na mata de t. firme, argilosa; flôres amarelas.
Fagara djalma-batistae Albuq.

- Publ. INPA, Botânica 27: 9-10; Tab. 7-8 (1968).

A coleção INPA 14778 Parátipo, consta de apenas um exemplar em nosso herbário, e um outro no herbário de NA, com os seguintes dados de coleta: Proc. Amazonas, Manaus, Reserva Florestal Ducke, picada base do levantamento / Leg. W. Rodrigues et A. Loureiro, 5826 / Em 4.VI. 1964 / Obs. Arbusto de $3 \mathrm{~m}$; frutos ainda verdes com odor de laranja.

Fagara prancei Albuq.

- Bol. INPA, Pesq. Flor. 8; 4pp; Fig. 1. (1970)

A coleção INPA 24686 consta de dois exemplares: o Holótipo, e um Isótipo, com exsicatas nos herbários de MG, NY, R e US. Em nossos exemplares constam os seguintes dados de coleta : Proc. Amazonas, caminho da margem leste do rio Yaco, até o rio Purus, $3 \mathrm{~km}$ acima da confluência / Leg. G. T. Prance, 7887 / Em 5.X.1968 / Obs. Árvore de $20 \mathrm{~m} \times 40 \mathrm{~cm}$ de diâmetro; mata de t. firme.

\section{SABIACEAE}

Phoxanthus manausensis W. Rodr.

- Publ. INPA, Botânica 17; 7pp.; 2 estps. (1964).

Nossa coleção referente à espécie acima consta do Holótipo, Isótipo (INPA 12954), e Parátipo (INPA 13194), com exemplares nos herbários de MG e IPEAN, respectivamente.

Da coleção (INPA 12954) temos o Holótipo e um Isótipo, com os seguintes dados de coleta: Proc. Amazonas, Manaus, $\mathrm{km} 7$ da estrada Manaus-Itacoatiara, Igarapé do Buião / Leg. W. Rodrigues et J. Chagas, 4463 / Em 24.V.1962 / Obs. Arvoreta de 6m na margem alagada do igarapé, solo arenoso; inflorescência rósea. 
Da coleção INPA 13194 consta apenas um exemplar em nosso herbário, com duplicatas nos herbários do IPEAN e MG. O nosso exemplar contém os seguintes dados de coleta: Proc. Amazonas, Manaus, Igarapé do Buiāo / Leg. W. Rodrigues et J. Chagas, 4680 / Em 5.X.1962 / Obs. Arvoreta de $6 \mathrm{~m}$ à beira alagada do igarapé. Frutos verdes, quando maduros roxo-escuros. Frutos maduros colhidos em 12.II. 63 .

\section{SAPOTACEAE}

Ecclinusa bacuri Aubr. et Pellegr.

- Adansonia I (2) : 21-22; Pl.6 (1, 2, 3, 4) (1961)

O exemplar INPA 1094 é Isótipo, com uma única exsicata no herbário do IPEAN e outra em nosso herbário. Nossa exsicata contém as seguintes indicações de coleta: Proc. Amazonas, Manaus, estrada do Mindu / N. V. Cauchorana / Leg. L. Coêlho, s.n. / Em. 30.V.1955 / Obs. Árvore de $20 \mathrm{~m}$ bastante esgalhada; mata de $t$. firme, úmida; látex branco.

Nota - O autor cita em seu trabalho que o espécime Tipo está em nossa coleção, porém o mesmo ainda não nos foi devolvido.

Micropholis rosadinha-brava Aubr. et Pellegr.

- Adansonia I (2): 178; Pl. 9 (10, 11, 12, 13, 14). (1961).

Nossa coleção referente à espécie acima consta dos Parátipos (INPA 5155 e 5548) com um exemplar em nosso herbário e outro no IPEAN respectivamente.

Em nosso herbário, os exemplares citados possuem as seguintes indicações de coleta : Coleção INPA 5155 - Proc. Amazonas, Manaus, Reserva Florestal Ducke / N. V. Rosada brava / Leg. L. Coêlho, s.n. / Em 14.III. 1957 Obs. Arvore de $16 \mathrm{~m}$; látex branco; t. firme, solo argiloso. (Material estéril).

Coleção INPA 5548 - Proc. Amazonas, Manaus, Reserva Florestal Ducke, A-723 / N. V. Rosada brava / Leg. B. Wilson, s.n. / Em 9.IV.1957 / Obs. Árvore; látex alvo; t. firme, solo arenoso-humoso. (Material estéril).

Nota - O autor cita no trabalho que o espécime Tipo (INPA 1097) está em nosso herbário, porém o mesmo ainda não nos foi devolvido, consequentemente em nossa coleção figuram apenas os Parátipos acima.

Micropholis williamii Aubr. et Pellegr.

- Adansonia I (2) : 179; Pl. 9 (1, 2, 3, 4, 5). (1961) .

A coleção INPA 7613 consta apenas de Isótipos, com exemplares nos herbários do INPA, IPEAN e NY. Em nosso herbário há apenas uma exsicata, com os seguintes dados de coleta : Proc. Amazonas, Manaus, Reserva Florestal Ducke / Leg. W. Rodrigues, D. Coêlho, 1284 / Obs. Árvore de $12 \mathrm{~m}$ de altura; mata de t. firme, solo argiloso; flôres amareladas.

Nota - Seg. o autor, o espécime Tipo encontra-se em nosso herbário, porém o mesmo ainda não nos foi restituido.

Richardella manausensis Aubr. et Pellegr.

- Adansonia I (2) : 176. (1961)

Da coleção INPA 7305 só possuimos um exemplar Isótipo em nosso herbário, com os seguintes dados de coleta: Proc. Amazonas, Manaus, Cachoeira Baixa do Tarumã / N. V. Abiurana mansa / Leg. L. Coêlho, s.n. / Em 30.IV. 1959 / Obs. Árvore de $20 \mathrm{~m}$; frutos vermelhos; mata de t. firme, solo arenoso (Foto 10) .

Nota - No trabalho original o autor diz que o espécime Tipo encontra-se em nosso herbário, porém o mesmo ainda não nos foi devolvido. 


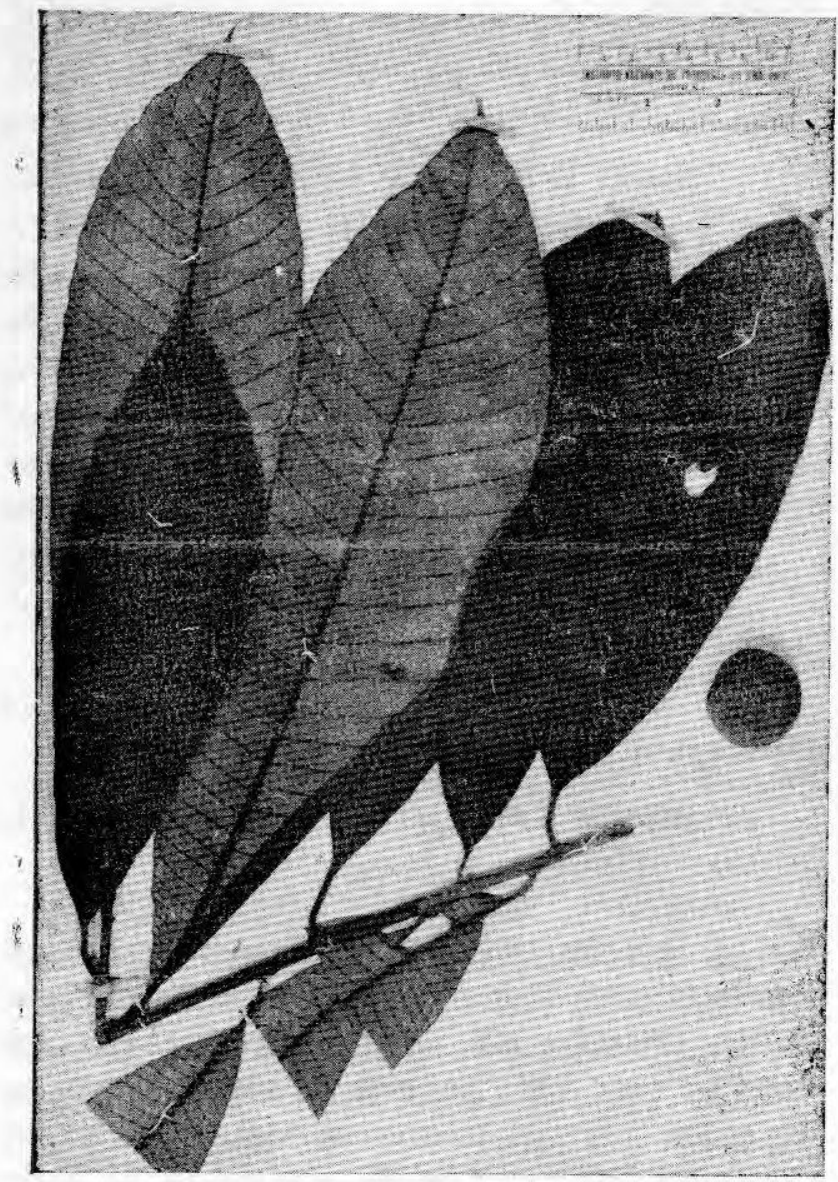

Foto 10 - Richardella manausensis

\section{VOCHYSIACEAE}

Erisma djalma-batistae: Paula

- Bol. Mus. Par. Emílio Goeldi. Bot. 28 : 2; Fıgs. 1, 2, 3, 4, 5, 6, 7, 8, 9, 10. (1967).

Nossa coleção referente à espécie acima consta de Holótipo, Isótipos (INPA 13472) com exemplares nos herbários do INPA e MG, e Parátipo (INPA 13657) no herbário do INPA.

Da coleção de referência INPA 13472 temos três exemplares em nosso herbário, o Holótipo e dois Isótipos, contendo as seguintes indicações de coleta: Proc. Amazonas, Manaus, Cachoeira Baixa do Tarumã / Leg. W. Rodrigues et D. Coêlho, 4940 / Em 26.XII. 62 / Obs. Árvore de $5 \mathrm{~m}$ de altura com flores amarelas; t. arenoso, úmido.

A coleção INPA 13657 consta de apenas um exemplar em nosso herbário, com as seguintes indicações de coleta: Proc. Amazonas, Manaus, Cachoeira Baixa do Tarumã / Leg. W. Rodrigues, 4996 / Em 13.II.1963 / Obs. Árvore de $7 \mathrm{~m}$ no bosque de solo arenoso; frutos esverdeados.

\section{SUMMARY}

This paper is a first publication of typus from Brazilian Amazonia available in the herbarium of Brazil's National Amazonian Research Institute (I.N.P.A.).

To complete herbarium material indications, collection data from hebarium labels were transcribed. shown.

10 photographs of not yet illustred types are

\section{BIBLIOGRAFIA CITADA}

Travassos, O. P.

1962/65 - Typus do Herbário do Jardim Botânico do Rio de Janeiro. Arq. Jard. Bot. do Rio de Janeiro, $18: 240-267$. 